\title{
Combined effects of EGFR and Hedgehog signaling pathway inhibition on the proliferation and apoptosis of pancreatic cancer cells
}

\author{
CHANG-FU QIN, KUN HAO, XIAO-DONG TIAN, XUE-HAI XIE and YIN-MO YANG \\ Department of General Surgery, Peking University First Hospital, Beijing 100034, P.R. China
}

Received January 19, 2012; Accepted March 12, 2012

DOI: $10.3892 /$ or.2012.1808

\begin{abstract}
In the present study, we established a new experimental model to investigate the effects of EGFR targeting by RNAi, and the synergistic actions between the Hedgehog (Hh) and EGFR signaling pathways on the proliferation and apoptosis in pancreatic cancer cells. Three human pancreatic cancer cell lines expressing EGFR shRNA were established, and gene expression inhibition was assessed in these lines using RT-PCR and western blot analysis. The effects of EGFR RNAi and Hh inhibition on cell proliferation and apoptosis were explored in vitro and in vivo. We observed that EGFR RNAi notably inhibited cell proliferation and colony formation, induced apoptosis and markedly decreased xenograft tumor growth. Furthermore, EGFR RNAi significantly enhanced cyclopamine sensitivity both in vitro and in vivo, and a synergistic decrease of both AKT and ERK phosphorylation was observed. The present study demonstrates that combined inhibition of both EGFR and Hh signaling pathways could establish a more promising antitumor approach than inhibiting each singly, and that there is a possible synergistic effect for Hh and EGFR signaling pathways on ERK and AKT phosphorylation.
\end{abstract}

\section{Introduction}

Pancreatic cancer is one of the most aggressive malignancies in the world, with a poor prognosis and a high mortality rate. The overall 5-year survival rate for pancreatic cancer patients remains less than 5\%. Although surgical approaches have been developed, there has been no significant improvement in survival rate over the past three decades (1), which is attrib-

Correspondence to: Professor Yin-Mo Yang, Department of General Surgery, Peking University First Hospital, 8th Xishiku Street, Xicheng District, Beijing 100034, P.R. China

E-mail: yangyinmo@263.net

Key words: RNAi, epidermal growth factor receptor, Hedgehog, cyclopamine, xenograft uted to the high incidence of metastasis at initial diagnosis (2). Further understanding of tumorigenesis of pancreatic cancer may provide new clues for developing prevention and treatment strategies.

Epidermal growth factor receptor (EGFR) plays a key role in epithelial tumor formation. Recent studies indicated that EGFR was detectable in over $95 \%$ of pancreatic cancer patients (3), and that aberrant EGFR activation can increase pancreatic cancer cell proliferation (4). In recent years, EGFR targeting therapy has become a popular mode of comprehensive tumor treatment. However, selective targeting of EGFR was not as effective as expected, because drug resistance would develop and lead to unsatisfactory clinical effect (5-7). Therefore, it remains necessary to explore additional therapeutic combinations.

Hedgehog (Hh) signaling pathway plays an important role in pancreatic carcinogenesis. It is closely associated with pancreatic cancer occurrence and progression, and is involved in cellular proliferation and invasion in vivo and in vitro (8). Research showed that EGFR and Hh signaling pathways were upregulated in many pancreatic cancer cell lines $(9,10)$. However, the relationship and the synergetic mechanisms between these two pathways remain unclear.

In this study, we introduced a lentiviral vector containing shRNA that targets the EGFR gene into human pancreatic cancer cells. The effects of EGFR RNAi alone or in conjunction with $\mathrm{Hh}$ inhibition on proliferation and apoptosis were explored both in vitro and in vivo, and the possible synergistic mechanisms for $\mathrm{Hh}$ and EGFR signaling pathways were further investigated.

\section{Materials and methods}

Cell line and culture conditions. The human pancreatic cancer cell lines, PANC-1, ASPC-1 and Mia PaCa-2 were generously provided by Professor Beger and Professor Kornmann of Ulm University, Germany. The cells were cultured in Dulbecco's modified Eagle's medium (DMEM, Gibco, Invitrogen, Carlsbad, CA, USA) supplemented with $10 \%$ fetal bovine serum (FBS, Sigma, St. Louis, MO, USA) and penicillin (100 U/ml).

Establishment of pancreatic cancer cell clones expressing EGFR RNAi. The RNAi targeting human EGFR sequence 
(GeneBank accession no. NM_201283) and the negative control sequence were designed and constructed by GeneChem (Shanghai, China). PANC-1 cells were transfected with recombinant $\mathrm{pFU}-\mathrm{GW}-\mathrm{RNAi}$ lentivirus vectors targeting the EGFR gene (PANC-1-si) or with negative control vectors (PANC-1-nc). All constructs were confirmed using sequence analysis. The constructs were stably transfected into cells to generate knockdown clones. The cells were cultured for $48 \mathrm{~h}$, and the transduction efficiency was assessed using FACS analysis. The transfected cells were harvested and prepared for subsequent studies.

Reverse transcriptase polymerase chain reaction (RT-PCR). Total RNA from wild-type pancreatic cancer cells and clones EGFR knockdown cells was extracted using TRIzol reagent (Invitrogen, Carlsbad, CA, USA). Aliquots ( $1 \mu \mathrm{g})$ of RNA were DNase-treated and processed for first-strand cDNA synthesis using the RT-PCR Kit (Toyobo, Osaka, Japan). cDNA was amplified using a PCR thermal controller with initial denaturation at $94^{\circ} \mathrm{C}$ for $5 \mathrm{~min}$, followed by 30 cycles of: denaturation at $94^{\circ} \mathrm{C}$ for $30 \mathrm{sec}$, annealing at $61^{\circ} \mathrm{C}$ for $30 \mathrm{sec}$, extension at $72^{\circ} \mathrm{C}$ for $60 \mathrm{sec}$, and a final extension step at $72^{\circ} \mathrm{C}$ for $5 \mathrm{~min}$. GAPDH was used for gene expression normalization. Three independent experiments were performed.

Western blot analysis. The total protein from wild-type pancreatic cancer cells and the EGFR knockdown clones (PANC-1, PANC-1-nc and PANC-1-si) were obtained using the Total Protein Extraction kit (Keygen, Nanjing, China). Total protein per sample $(70 \mu \mathrm{g})$ was separated by sodium dodecyl sulfate-polyacrylamide gel electrophoresis (SDS-PAGE) and electroblotted onto nitrocellulose membranes, and then probed with anti-phospho-Akt, anti-Akt, anti-phospho-Erk1/2, anti-Erk1/2 (Cell Signaling Technology, Beverly, MA, USA) and anti-EGFR (Abcam, Cambridge, UK) antibodies. Three independent experiments were performed.

Colony formation assay. To assess the effect of EGFR knockdown on the colony formation ability of PANC-1, PANC-1-nc and PANC-1-si cells, each were respectively seeded onto 6 -well plates at various low densities. When clones appeared, they were fixed in methanol and stained with Giemsa before they were manually counted. Three independent experiments were performed.

Cell growth assays. The MTT assay was used to assess the effect of EGFR knockdown on cell proliferation and cyclopamine sensitivity in pancreatic cancer cells. Wild-type cancer cells (PANC-1) and two transduced clones (PANC-1-nc and PANC-1-si) were cultured in 96-well plates at a density of $1.0 \times 10^{4}$ cells/well for $72 \mathrm{~h}$ prior to the proliferation assay. To assess cyclopamine sensitivity, each cell group was seeded at a density of $1.0 \times 10^{4}$ cells/well in 96-well plates, and allowed to attach for $24 \mathrm{~h}$. They were then incubated in media containing the indicated concentrations $(0,0.625,1.25,2.5,5.0$ and $10 \mu \mathrm{mol} / \mathrm{l})$ of cyclopamine for $48 \mathrm{~h}$ before initiation of the MTT assay. The cell growth inhibition rate (GIR) was calculated as follows: $\mathrm{GIR}=\left(1-\mathrm{OD}_{490}\right.$ of treated cells $/ \mathrm{OD}_{490}$ of untreated cells) x $100 \%$. Three independent experiments were performed.

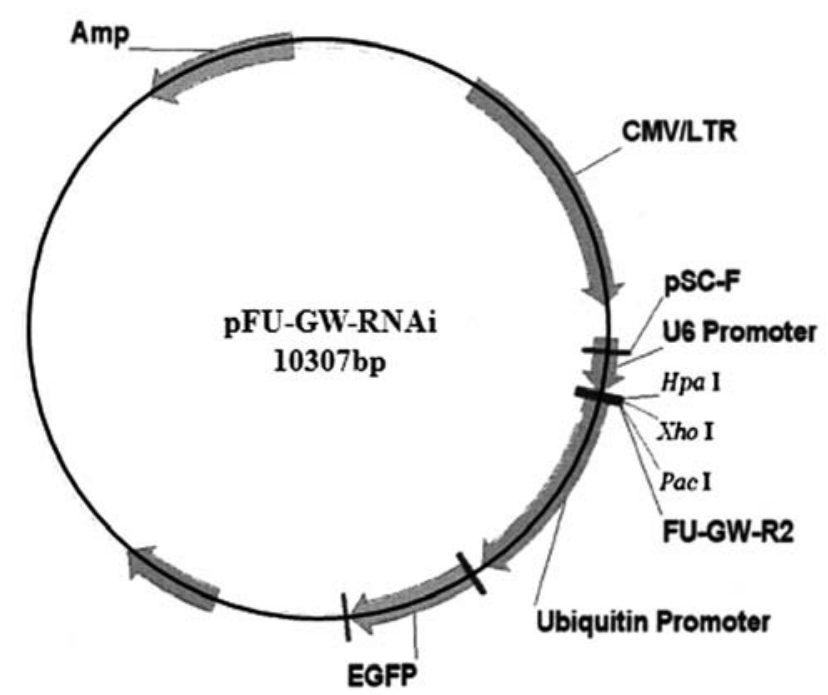

Figure 1. Schematic diagram of the pFU-GW-RNAi vector. The shRNAencoding template was inserted between the $\mathrm{HpaI}$ and $\mathrm{XhoI}$ restriction sites downstream of U6 promoter.

Flow cytometry analysis for apoptosis. An Annexin V-PE Apoptosis Detection kit (BD Biosciences) was used to measure apoptosis in PANC-1, PANC-1-nc and PANC-1-si cells, with or without cyclopamine treatment. The cells were seeded in 6-well plates and incubated until they were about $70 \%$ confluent. Then, the cyclopamine medium was replaced with fresh media, and the cells were incubated for $48 \mathrm{~h}$ before analysis. The labeled cells $\left(1.0 \times 10^{4} / \mathrm{sample}\right)$ were analyzed using FACScan flow cytometry (BD Biosciences) in conjunction with CellQuest software. Three independent experiments were performed.

Tumor xenografts in nude mice. All animal studies were reviewed and approved by the Ethics Committee for Animal Studies at Peking University, China. Briefly, $1.0 \times 10^{7}$ cells were injected subcutaneously into the right axilla of 6-week-old female nude mice. The mice were randomized into four treatment groups according to the cells injected and paired with the following treatments: i) PANC-1-nc, or PANC-1-si plus cyclopamine $(50 \mathrm{mg} / \mathrm{kg}$, dissolved in $0.1 \mathrm{ml}$ PBS, intraperitoneal injection, once every other day) (11) treatment, and ii) PANC-1-nc, or PANC-1-si plus PBS treatment groups $(0.1 \mathrm{ml}$ intraperitoneal injection, once every other day). The animals were monitored for tumor formation every 2 days. Tumor size was measured in two dimensions, and the volume was calculated using the equation $\mathrm{V}=\mathrm{L} \times \mathrm{W}^{2} \times 0.5$ (where $\mathrm{V}$ is the volume, $\mathrm{L}$ is the length and $\mathrm{W}$ is the width). Four weeks later, all the mice were sacrificed, and the tumors were excised and weighed.

Immunohistochemistry analysis of xenograft tumors. Paraffinembedded $4-\mu \mathrm{m}$-thick sections were prepared and analyzed by H\&E staining or immunohistochemical analysis using a Cell and Tissue Staining kit according to the manufacturer's protocol (R\&D Systems, Minneapolis, MN, USA). To assess the proliferative index of serial sections, Ki-67 staining assay was carried out in representative non-necrotic areas of each 

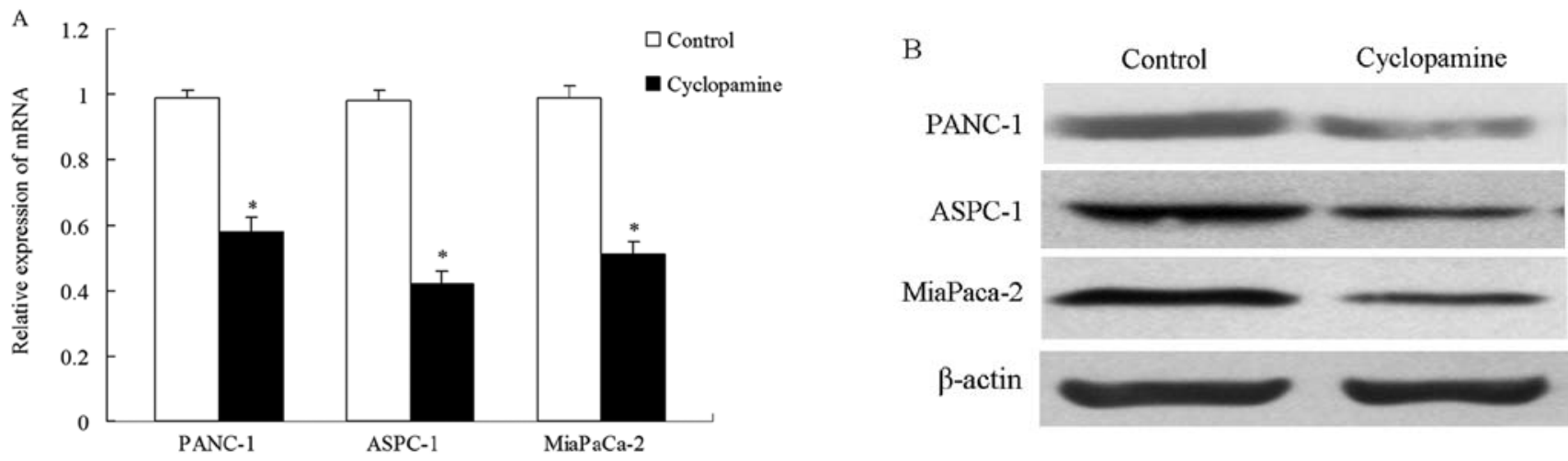

Figure 2. Cyclopamine blocks EGFR expression in pancreatic cancer cell lines. (A) EGFR mRNA expression was detected using real time-PCR. Results were expressed as mean $\pm \mathrm{SD}$ from three experiments ( $\left.{ }^{*} \mathrm{P}<0.05\right)$. The error bars represent the standard error of the mean $(\mathrm{SEM})$. (B) EGFR protein expression was analyzed using western blot analysis. $\beta$-actin served as a loading control.

A

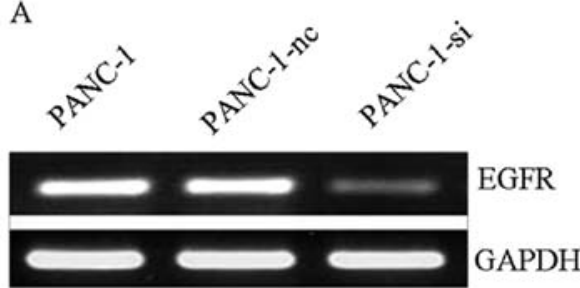

B

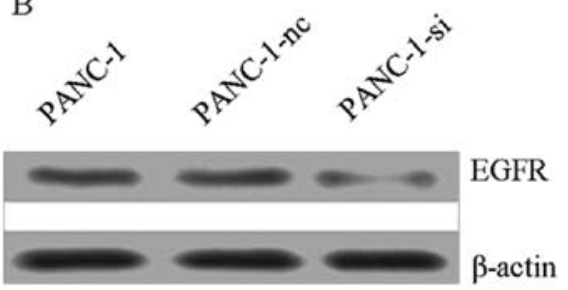

Figure 3. EGFR expression in cultured pancreatic cancer cell lines (PANC-1, PANC-1-nc and PANC-1-si). (A) EGFR mRNA expression detected using RT-PCR. (B) Protein expression of EGFR detected using western blot analysis. GAPDH and $\beta$-actin served as loading controls.

tumor using a rabbit anti-human Ki-67 antibody (Abcam). Tumor cell apoptosis was evaluated using the TUNEL assay (Roche, Mannheim, Germany) and was performed as described by the manufacturer.

Statistical analysis. Quantitative data were expressed as means \pm SD. Means were compared using one-way ANOVA and Student's t-test. Statistical analyses were performed using SPSS Version 13.0. Differences were considered statistically significant if $\mathrm{P}<0.05$.

\section{Results}

Detection of endogenous EGFR in pancreatic cancer cell lines and the effect of cyclopamine on its expression. EGFR mRNA and protein expression were determined using RT-PCR and western blot analyses, respectively, in three pancreatic cancer cell lines (PANC-1, ASPC-1 and Mia PaCa-2). As shown in Fig. 2A, the EGFR mRNA and protein expression levels differed in the three cell lines. We then studied the effect of cyclopamine, a naturally occurring Hh pathway antagonist, on EGFR expression in these cell lines. As shown in Fig. 2B, both protein and mRNA expression levels decreased in all the three cell lines after $48 \mathrm{~h}$ of treatment with $5 \mu \mathrm{mol} / 1$ cyclopamine. EGFR mRNA and protein expression in PANC-1 cells was higher than in the other two cell lines ( $\left.{ }^{*} \mathrm{P}<0.05\right)$. Therefore, we selected the PANC-1 cell line for follow-up assays in order to show EGFR inhibition more clearly.
RNAi targeting EGFR in PANC-1 pancreatic cancer cells. PANC-1 cells were transfected with either recombinant pFU-GW-RNAi lentiviral vectors that target the EGFR gene (PANC-1-si), or with negative control vectors (PANC-1-nc). The stably transfected cells were purified using FACS. RT-PCR and western blot analyses probed for EGFR expression and revealed that it was significantly downregulated in PANC-1-si transfected cells, but not in PANC-1-nc transfected cells when both were compared with untransfected cells (Fig. 3). The results indicated that the EGFR-targeting RNAi could effectively suppress EGFR gene expression in PANC-1 cells.

Combined targeting of EGFR and Hh signaling enhanced inhibition of proliferation and colony formation in PANC-1 cells. The effect of EGFR inhibition on cell growth was measured using the MTT and colony formation assays. Colony formation assay was used to assess cell survival, as shown in Fig. 4. The results indicated that the number of PANC-1-si+cyclopamine colonies was fewer than those of PANC-1-nc cells and PANC-1-si cells ( $\mathrm{P}<0.05$, Fig. 4). There was no difference in colony formation inhibition when PANC-1-nc+cyclopamine cells and PANC-1-si cells were compared $(\mathrm{P}>0.05)$.

Cell proliferation was analyzed using the MTT assay. Proliferation was inhibited notably for EGFR knockdown cells when combined with $\mathrm{Hh}$ signaling inhibition by cyclopamine in the PANC-1 cell line. As shown in Fig. 5A, cyclopamine inhibited cell growth in a dose-dependent manner. The inhibi- 


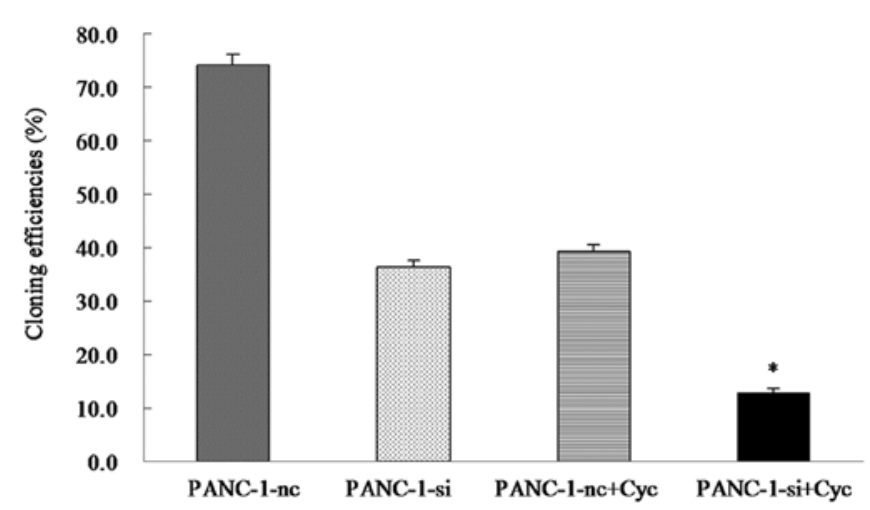

Figure 4. Colony formation efficiencies in different EGFR expression groups. The PANC-1-si had fewer colonies than PANC-1-nc and PANC-1 cell lines. Three independent experiments were performed. ${ }^{*} \mathrm{P}<0.05$. The error bars in the figures represent the standard error of the mean (SEM).

tory rates after $72 \mathrm{~h}$ of treatment were $32.67 \%$ for PANC-1, 9.03\% for PANC-1-nc and $6.99 \%$ for PANC-1-si cells. A low concentration of cyclopamine $(0.625 \mu \mathrm{mol} / \mathrm{l})$ was sufficient to significantly inhibit cell growth. PANC-1-si cells displayed the highest sensitivity to cyclopamine, due to EGFR knockdown. However, there was no difference in growth inhibition between the PANC-1 and PANC-1-nc cell lines $(\mathrm{P}>0.05)$. This result showed that treatment with cyclopamine resulted in a dosedependent anti-proliferative effect in PANC-1 cells. The half maximal inhibitory concentration $\left(\mathrm{IC}_{50}\right)$ for cyclopamine was determined from dose-response curves. As shown in Fig. 5B, the $\mathrm{IC}_{50}$ of cyclopamine was $2.978 \pm 0.336 \mu \mathrm{mol} / 1$ for PANC- 1 , $3.106 \pm 0.176 \mu \mathrm{mol} / \mathrm{l}$ for PANC-1-nc, and $1.698 \pm 0.057 \mu \mathrm{mol} / 1$ for PANC-1-si cells. The $\mathrm{IC}_{50}$ for the EGFR knockdown cells was significantly lower than those of wild-type and control transfected cells $(\mathrm{P}=0.002$ for $\mathrm{PANC}-1$ cells, $\mathrm{P}=0.001$ for PANC-1-nc cells and $\mathrm{P}=0.002$ for PANC-1-si cells). There was no difference in the $\mathrm{IC}_{50}$ between the control (PANC-1) and the untreated (PANC-1-nc) cells (P>0.05). These results showed that EGFR RNAi combined with Hh signaling inhibition could significantly reduce cell proliferation and colony formation in pancreatic cancer cells, and could effectively enhance cyclopamine sensitivity in vitro.

Synergetic effects of EGFR and Hh signaling inhibition on apoptosis in pancreatic cancer cells. Apoptosis of cultured cells was analyzed using flow cytometry. Four different conditions were tested: PANC-1-nc, PANC-1-si, PANC-1-nc+Cyc and PANC-1-si+Cyc. As shown in Fig. 6, the prophase apoptosis percentage of the control group (PANC-1-nc) was 3.117 $\pm 0.121 \%$ $(\mathrm{P}=0.001)$; and both the EGFR RNAi group (PANC-1-si) and the cyclopamine treatment group (PANC-1-nc+Cyc) showed a moderately increased apoptosis, compared with untreated PANC-1-nc cells $(\mathrm{P}<0.05)$. The prophase apoptosis percentage of the combined treatment group (PANC-1-si+Cyc) was as high as $38.97 \pm 1.237 \%$, which was significantly higher than the EGFR knockdown (PANC-1-si, 17.62 $\pm 0.602 \%$ ) and the control group (PANC-1-nc, $3.117 \pm 0.121 \%),(\mathrm{P}<0.05)$. The prophase apoptosis percentage of the combined treatment group (PANC-1-si+Cyc) was significantly higher than that of the PANC-1-si and PANC-1-nc+Cyc groups $(\mathrm{P}<0.05)$. The
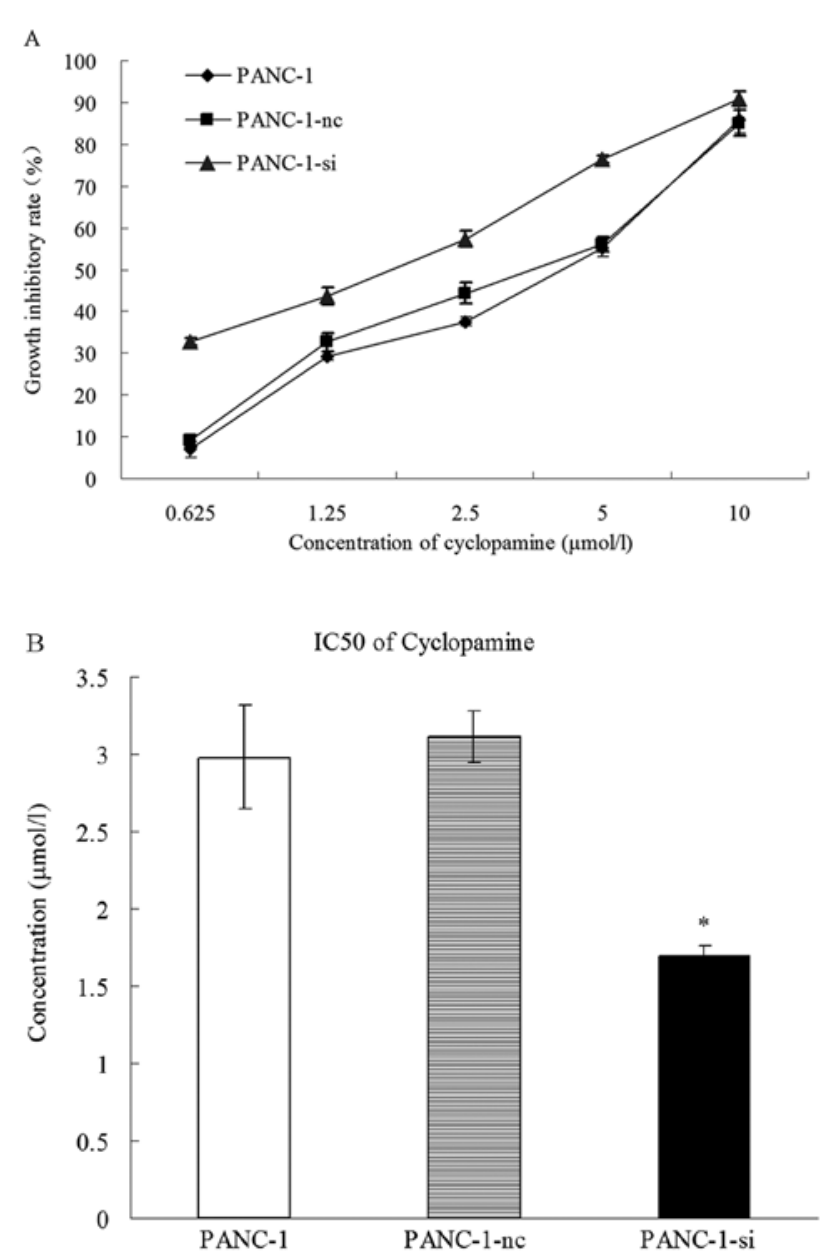

Figure 5. Effects of cyclopamine on the proliferation of pancreatic cancer cells and EGFR RNAi clones. (A) Growth inhibition of PANC-1, PANC1-nc, and PANC-1-si cells at different dosages of cyclopamine. (B) $\mathrm{IC}_{50}$ of cyclopamine on PANC-1, PANC-1-nc and PANC-1-si cells. " $\mathrm{P}<0.05$. The error bars in the figures represent the standard error of the mean (SEM).

results showed that EGFR downregulation might reduce the apoptosis threshold in pancreatic cancer cells, and enhanced apoptosis when combined with cyclopamine treatment.

Effects of EGFR RNAi and cyclopamine on PANC-1 xenograft growth. To investigate whether the in vitro antitumor effects of EGFR inhibition and its synergistic effects with cyclopamine could be reproduced in vivo, we established xenograft models using PANC-1-nc and PANC-1-si cells. All of the PANC-1-nc injected mice, and most of the PANC-1-si injected mice developed measurable tumors one week after injection. The mice then received treatments of either cyclopamine or PBS once every $48 \mathrm{~h}$. The xenograft tumor size was measured once a week. As shown in Fig. 7, the tumors from PANC-1-si injected mice were significantly smaller than tumors from the control cells (PANC-1-si+PBS vs. PANC-1-nc+PBS, P<0.05). Cyclopamine treatment could inhibit xenograft tumor growth compared with PBS (PANC-1-nc+Cyc vs. PANC-1-nc+PBS, $\mathrm{P}<0.05$ ). Furthermore, the combined treatment using EGFR RNAi and cyclopamine (PANC-1-si+Cyc) resulted in additional tumor growth inhibition (Fig. 7A). Twenty-eight days after tumor inoculation, the average tumor volume of the combined treatment was significantly decreased when 

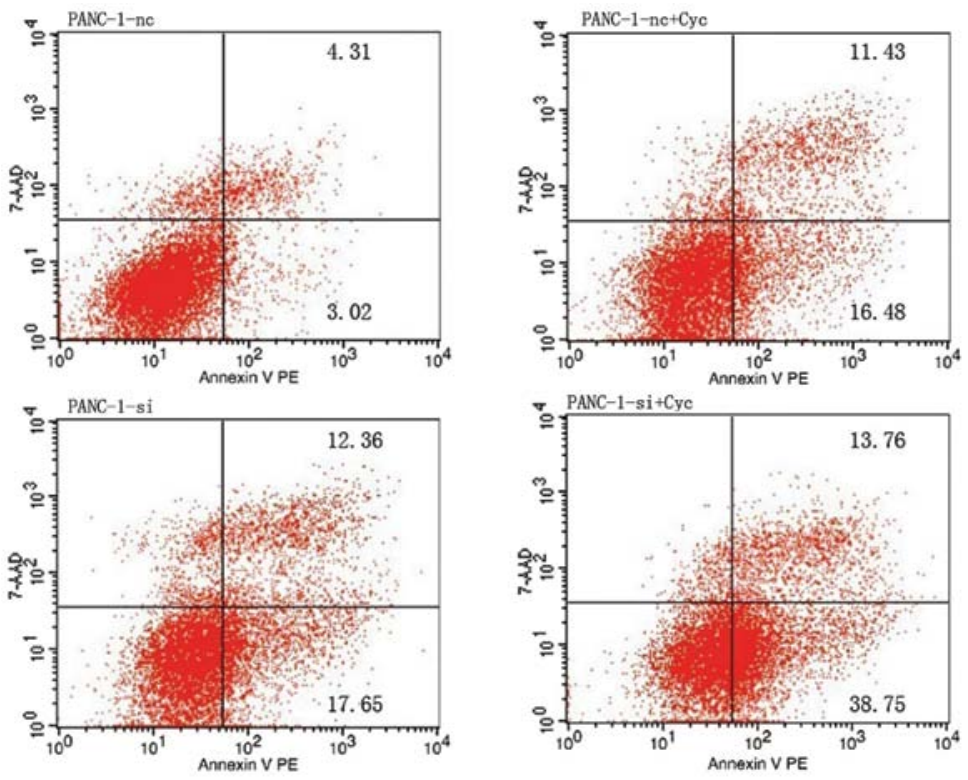

Figure 6. Apoptosis in pancreatic cancer cells as detected by flow cytometry. The average apoptosis rates of control group (PANC-1-nc), EGFR RNAi group (PANC-1-si), cyclopamine-treated group (PANC-1-nc+Cyc), and combined treatment group (PANC-1-si+Cyc) were 3.117 $\pm 0.121 \%, 17.62 \pm 0.602 \%$, $16.44 \pm 0.645 \%$, and $38.97 \pm 1.237 \%$, respectively.
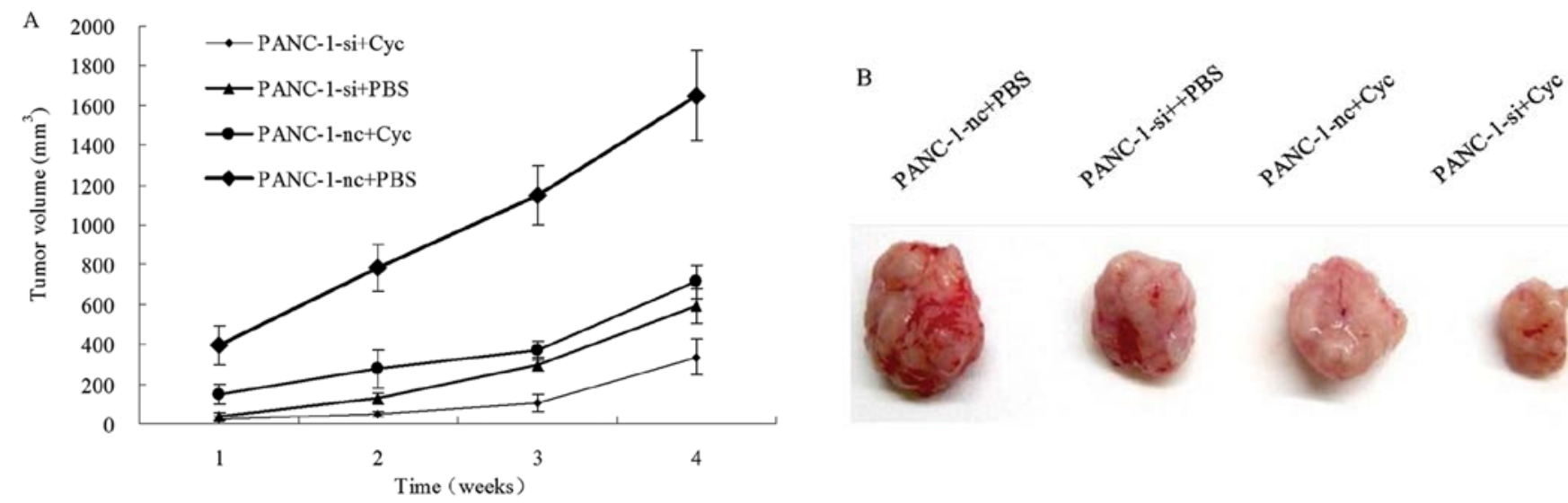

Figure 7. Synergistic effect of EGFR siRNA and cyclopamine on xenograft tumors growth. PANC-1-si or PANC-1-nc cells were subcutaneously injected into the right axilla of nude mice. (A) The tumor volume was monitored every 2 days when measurable tumors appeared after inoculation with PANC-1-nc and PANC-1-si cells. (B) Average tumor volume on Day 28. The average tumor volume of PANC-1-si+Cyc was notably smaller than those from the other three groups.

compared with those from PANC-1-nc+PBS PANC-1-si+PBS and PANC-1-nc+Cyc xenografts $(\mathrm{P}<0.05$, Fig. 7B).

Proliferation and apoptosis in xenograft tumors. We performed the Ki-67 staining assay and the TUNEL assay to evaluate proliferation in xenografts. As shown in Fig. 8, analysis of the proliferative index for the xenograft tumors revealed that both suppression of EGFR and combined treatment with cyclopamine significantly inhibited Ki-67 immunoreactivity, compared with the control groups. Combined treatment (PANC-1-si+Cyc xenografts) showed the lowest Ki-67 immunoreactivity. Tumor cell apoptosis was also assessed using the TUNEL assay (Fig. 8A). As shown in Table I, the apoptotic index for PANC-1-si+Cyc cells was significantly higher than PANC-1-nc cells and PANC-1-si cells $\left({ }^{*} \mathrm{P}<0.05\right)$. The results indicated that inhibition of both EGFR and Hh signaling pathways may have a synergistic effect on proliferation and apoptosis.

Synergistic effects of EGFR and Hh inhibition on PI3K/Akt and Raf/MEK/ERK activation. To investigate possible mechanisms for the synergistic apoptosis effect, we analyzed the PI3K/Akt and Raf/MEK/ERK activation using western blot analysis. As shown in Fig. 9, EGFR knockdown combined with cyclopamine-mediated $\mathrm{Hh}$ inhibition produced a pronounced decrease in both AKT and ERK phosphorylation, when compared with PANC-1-si or PANC-1-nc+Cyc cells. Moreover, phosphorylated AKT/ERK expression in PANC-1-si+Cyc tumor groups displayed a significant reduction compared with the control group (Fig. 10). 


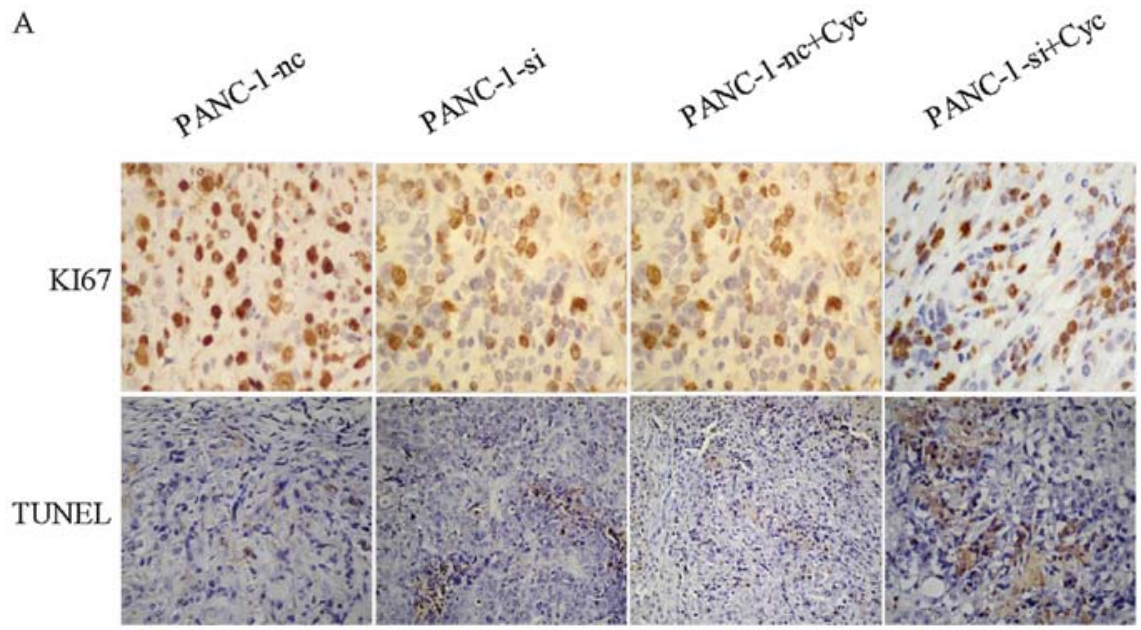

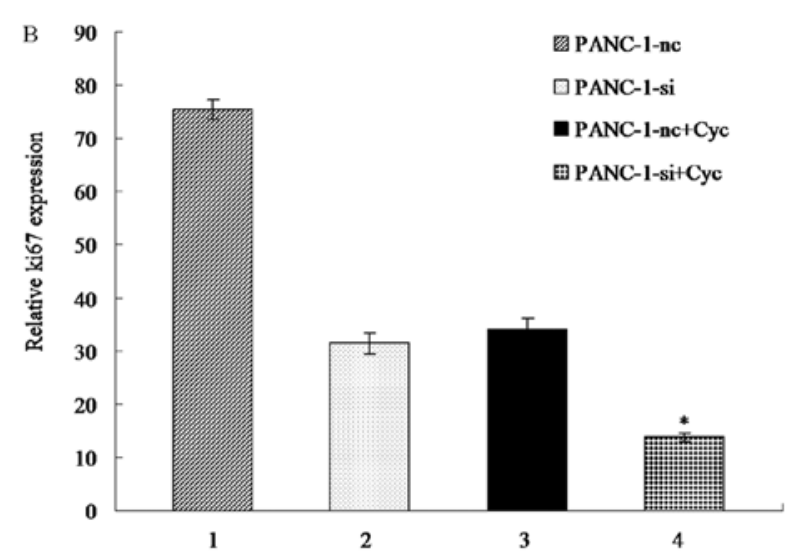

Table I. Apoptotic index.

\begin{tabular}{lccr}
\hline Cells & $\begin{array}{c}\text { Control } \\
\text { (NS) }\end{array}$ & $\begin{array}{c}\text { Treatment group } \\
\text { (cyclopamine) }\end{array}$ & P-value \\
\hline PANC-1-nc & $3.85 \pm 0.52$ & $12.76 \pm 2.29$ & 0.003 \\
PANC-1-si & $14.73 \pm 1.00$ & $20.87 \pm 4.38^{\mathrm{a}}$ & 0.002 \\
\hline $\begin{array}{l}\text { The apoptotic index of PANC-1-si+Cyc cells was significantly } \\
\text { higher than the controls (P<0.05). }\end{array}$ \\
\hline
\end{tabular}

\section{Discussion}

The epidermal growth factor receptor (EGFR) belongs to a family of receptor tyrosine kinases, and plays a key role in cell proliferation, survival, migration and differentiation in epithelial tumor formation (12). EGFR is localized mainly to cell membranes and is activated by the EGF ligand (13). A recent study revealed that the overexpression or deregulation of EGFR induced rapid growth, strong invasion ability, and poor prognosis for many kinds of solid tumors, including pancreatic cancer (14).

In recent years, therapy targeting EGFR has been a popular method for comprehensive tumor treatment, which includes EGFR monoclonal antibodies and small molecular tyrosine kinase inhibitors. For example, erlotinib, an EGFR-tyrosine kinase inhibitor, has been approved by the FDA as a compre-
Figure 8. Evaluating proliferation and apoptosis in xenograft tumors. Immunohistochemistry of serial sections using a Ki-67 antibody displayed nuclear Ki-67 immunoreactivity. As shown in (B), the highest percentage of Ki-67 positive cell was observed in the PANC-1-nc group, $75.39 \pm 1.83 \%$, and the combination of EGFR RNAi and cyclopamine treatment (PANC-1si+Cyc) revealed the lowest Ki-67 positive percentage, $13.82 \pm 0.93 \%$, compared with the other groups, $\left({ }^{*} \mathrm{P}<0.05\right)$. The error bars in the figures represent the standard error of the mean (SEM). There were no significant differences between the PANC-1-nc+Cyc and PANC-1-si groups. As shown in (A) apoptotic nuclei appeared in the xenografts after different treatments, as judged by TUNEL staining. The apoptotic index was measured three times. As shown in Table I, the apoptotic index of PANC-1-si+Cyc cells was significantly higher than the controls $(\mathrm{P}<0.05)$. The results are expressed as the mean of three experiments (mean $\pm \mathrm{SD} \%, \mathrm{n}=3$ ).

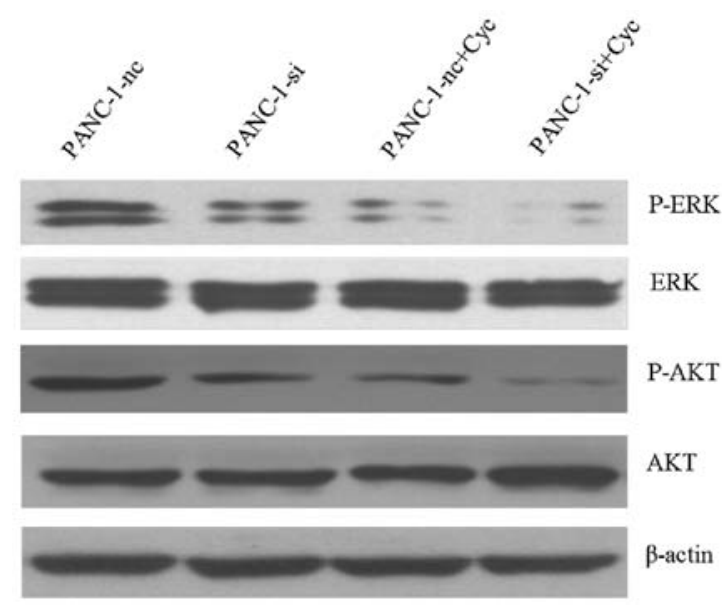

Figure 9. Synergistic effect of combined EGFR RNAi and cyclopamine treatment on Akt and ERK activation. Total cellular proteins (70 $\mu \mathrm{g})$ from the indicated cells were subjected to western blot analysis with antibodies directed against total or phosphorylated AKT/ERK. $\beta$-actin served as loading control. Representative results of three independent experiments are shown.

hensive treatment for advanced pancreatic cancer. However, monotherapy that targets EGFR has not been as effective as expected, and the clinical effect remains unsatisfactory. Patients with advanced pancreatic cancer have a poor prognosis and there have been no improvements in survival since the introduction of gemcitabine as a therapeutic option. A randomized phase III trial showed that improvement in patient 


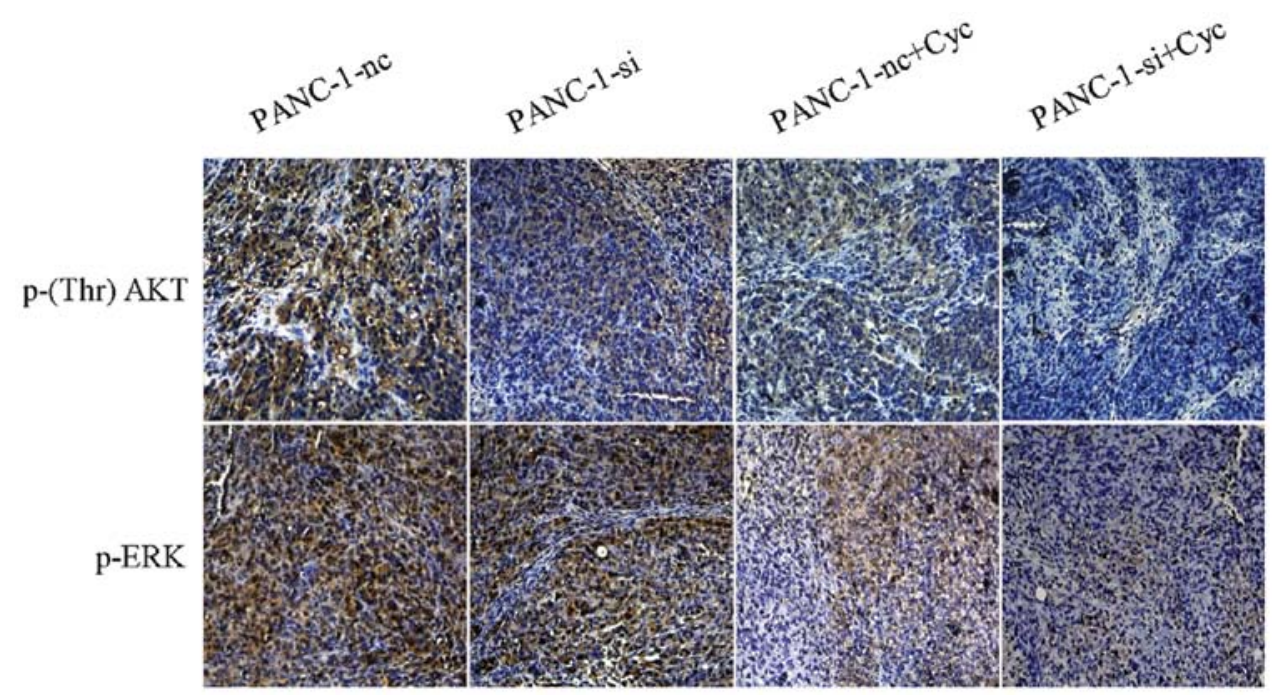

Figure 10. Immunohistochemical analysis of phosphorylated AKT/ERK in xenograft tumors. The expression of phosphorylated AKT/ERK in PANC-1-si+Cyc tumor groups displayed a significant reduction compared with the control group.

survival using erlotinib or gemcitabine singly was modest $(6.24$ vs. 5.91 months), while the 1-year survival rate using erlotinib combined with gemcitabine was 23 vs. $17 \%$ when combining placebo with gemcitabine, a statistically significant difference (6). Therefore, there is an urgent need to explore the crosstalk mechanism between EGFR and other signaling pathways to find a more efficient combinatory antitumor strategy.

The Hh signaling pathway contributes to pancreatic cancer occurrence and progression, and participates in maintaining the biological characteristics of pancreatic cancer stem cells (15). Upregulated Hh pathway activity has been observed in human pancreatic cancer $(16,17)$. Inhibition of Hh signaling by cyclopamine, a smoothened antagonist, inhibited pancreatic cancer growth in vitro and in vivo, suggesting that this signaling pathway has an early and critical role in pancreatic cancer formation (18). Specifically blocking Hh signaling can inhibit cell proliferation and invasion in vivo and in vitro, and may become one of the best hopes for targeted pancreatic cancer therapy. Some scholars have demonstrated a synergy between Hh and EGFR pathways in pancreatic cancer cell lines (19). Researchers have found that sonic Hedgehog (SHH) and EGFR positivity were significantly higher in 49 pancreatic cancer specimens compared with 49 matched normal specimens, as detected by immunohistochemistry (79.6 vs. $14.3 \%$, 73.5 vs. $16.3 \%, \mathrm{P}<0.05$ ), and $\mathrm{SHH}$ expression was positively correlated to EGFR expression $\left(r_{1}=0.232, \mathrm{P}<0.05\right)(9)$. Another study showed that there was an additive effect between anti-Hh and anti-EGFR pathways that enhanced sensitivity to antitumor drugs in esophageal and prostate cancer cell lines (20). Hu et al (21) found that Hh and EGFR were overexpressed in pancreatic cancer cell lines and the antitumor effects of Iressa, a tyrosine kinase inhibitor, were enhanced by downregulation of EGFR gene expression while blocking Hh signaling with cyclopamine.

In this study, we confirmed that EGFR was expressed in pancreatic cancer cell lines, and its expression was suppressed to some extent by cyclopamine. Our results indicate that EGFR knockdown could efficiently inhibit cell proliferation, increase apoptosis, and enhance cyclopamine sensitivity in human pancreatic cancer cells. EGFR downregulation significantly lowered the apoptotic threshold and enhanced sensitivity to cyclopamine. Cyclopamine appeared to selectively induce apoptosis in tumor cells without adverse effects to normal tissues in vivo (22), which suggests cyclopamine is a promising drug for preventing pancreatic cancer progression. Compared with singly blocking EGFR expression, the addition of cyclopamine to EGFR knockdown further inhibited cell proliferation and tumor growth in both cell lines and xenografts. The combinational treatment inhibited pancreatic cancer cell proliferation and colony formation, and effectively enhanced cyclopamine sensitivity in vitro. These results point to a synergistic effect between the Hh and EGFR signaling pathways in the regulation of cell proliferation and apoptosis. However, the mechanism between the two pathways remains unclear.

Two important downstream signaling pathways are controlled by EGFR. The first is the RAS-RAF-MEK-MAPK pathway, which influences gene transcription and cell proliferation. The second is the PI3K-Akt pathway, which regulates apoptosis resistance and survival signals $(23,24)$. They might also be involved in pancreatic cancer cell resistance to EGFRtargeted therapy $(25,26)$. In the Ras-Raf-MEK-ERK/MAPK pathway, ERK plays a dual role as a membrane protein and as a transcription factor. Activated MAPKs phosphorylate and regulate specific intranuclear transcription factors (27), thus inducing cell differentiation and proliferation that contribute to tumorigenesis and migration (28). AKT is a protooncogene, coding for a serine/threonine protein kinase (29), which is activated by phospholipid binding and activation loop phosphorylation at Thr308 by PDK1 $(30,31)$, and by phosphorylation within the C-terminus at Ser473 (32). AKT promotes cell survival by phosphorylating and inactivating apoptosis-inducing targets, including Bad (33), forkhead transcription factors (34) and caspase-9 (35). Since ERK and AKT are closely linked to cell survival and growth, we analyzed ERK and AKT phosphorylation using western blot analysis. The result showed that combining RNAi-silenced EGFR with Hh signal inhibition produced a marked decrease in both AKT and ERK phosphorylation in vivo and in vitro. The synergistic 
effect of dual EGFR and Hh signaling inhibition on proliferation and apoptosis, as presented in this study, suggests that combined treatment is likely to be a more efficient antitumor strategy than inhibiting either signal alone.

In conclusion, our present study showed that the $\mathrm{Hh}$ signaling pathway is involved in regulating EGFR expression, and point to a synergistic effect of Hh and EGFR. Hh pathway inhibition enhanced the effect of selective EGFR targeting on cell proliferation, and increased apoptosis in human pancreatic cancer cells in vivo and in vitro. Silencing EGFR in combination with cyclopamine may be a potential therapeutic strategy that significantly reduces tumor size and induces apoptosis. The synergistic mechanism of Hh and EGFR signaling pathways partly contributed to ERK and AKT phosphorylation. Although results have been encouraging, additional studies are warranted.

\section{Acknowledgements}

This research was supported by grants from the National Natural Science Foundation of China (no. 30972897 to Y.-M.Y). Thanks to Professor Ze-Bin Mao of the Department of Biochemistry and Molecular Biology in Peking University Health Science Center for his assistance and technical support.

\section{References}

1. Jemal A, Siegel R, Ward E, et al: Cancer statistics, 2006. CA Cancer J Clin 56: 106-130, 2006.

2. Kornmann M, Beger HG and Link KH: Chemosensitivity testing and test-directed chemotherapy in human pancreatic cancer. Recent Results Cancer Res 161: 180-195, 2003.

3. Papageorgio C and Perry MC: Epidermal growth factor receptortargeted therapy for pancreatic cancer. Cancer Invest 25: 647-657, 2007.

4. Yarden Y: The EGFR family and its ligands in human cancer. signalling mechanisms and therapeutic opportunities. Eur J Cancer 37 (Suppl 4): S3-S8, 2001.

5. Kelley RK and Ko AH: Erlotinib in the treatment of advanced pancreatic cancer. Biologics 2: 83-95, 2008.

6. Moore MJ, Goldstein D, Hamm J, et al: Erlotinib plus gemcitabine compared with gemcitabine alone in patients with advanced pancreatic cancer: a phase III trial of the National Cancer Institute of Canada Clinical Trials Group. J Clin Oncol 25: 1960-1966, 2007.

7. Rajput A, Koterba AP, Kreisberg JI, Foster JM, Willson JK and Brattain MG: A novel mechanism of resistance to epidermal growth factor receptor antagonism in vivo. Cancer Res 67: 665-673, 2007

8. Feldmann G, Dhara S, Fendrich V, et al: Blockade of hedgehog signaling inhibits pancreatic cancer invasion and metastases: a new paradigm for combination therapy in solid cancers. Cancer Res 67: 2187-2196, 2007.

9. Hu WG, Wang CY, Liu T, Xiong JX and Yang ZY: Expression of sonic hedgehog, EGFR and PCNA proteins in pancreatic cancer and their correlations to cell proliferation. Ai Zheng 26: 947-951, 2007 (In Chinese).

10. Ciardiello F and Tortora G: EGFR antagonists in cancer treatment. N Engl J Med 358: 1160-1174, 2008.

11. Berman DM,Karhadkar SS, Hallahan AR, et al: Medulloblastoma growth inhibition by hedgehog pathway blockade. Science 297: $1559-1561,2002$.

12. Jorissen RN, Walker F, Pouliot N, Garrett TP, Ward CW and Burgess AW: Epidermal growth factor receptor: mechanisms of activation and signalling. Exp Cell Res 284: 31-53, 2003.

13. Shi F, Telesco SE, Liu Y, Radhakrishnan R and Lemmon MA: ErbB3/HER3 intracellular domain is competent to bind ATP and catalyze autophosphorylation. Proc Natl Acad Sci USA 107: 7692-7697, 2010
14. Larsen AK, Ouaret D, El Ouadrani K and Petitprez A: Targeting EGFR and VEGF(R) pathway cross-talk in tumor survival and angiogenesis. Pharmacol Ther 131: 80-90, 2011.

15. Lee CJ, Dosch J and Simeone DM: Pancreatic cancer stem cells. J Clin Oncol 26: 2806-2812, 2008.

16. Berman DM, Karhadkar SS, Maitra A, et al: Widespread requirement for Hedgehog ligand stimulation in growth of digestive tract tumours. Nature 425: 846-851, 2003.

17. Thayer SP, di Magliano MP, Heiser PW, et al: Hedgehog is an early and late mediator of pancreatic cancer tumorigenesis. Nature 425: 851-856, 2003.

18. Chen JK, Taipale J, Cooper MK and Beachy PA: Inhibition of Hedgehog signaling by direct binding of cyclopamine to Smoothened. Genes Dev 16: 2743-2748, 2002.

19. Buck E, Eyzaguirre A, Haley JD, Gibson NW, Cagnoni P and Iwata KK: Inactivation of Akt by the epidermal growth factor receptor inhibitor erlotinib is mediated by HER-3 in pancreatic and colorectal tumor cell lines and contributes to erlotinib sensitivity. Mol Cancer Ther 5: 2051-2059, 2006.

20. Dragovich T and Campen C: Anti-EGFR-targeted therapy for esophageal and gastric cancers: an evolving concept. J Oncol 2009: 804108, 2009.

21. Hu WG, Liu T, Xiong JX and Wang CY: Blockade of sonic hedgehog signal pathway enhances antiproliferative effect of EGFR inhibitor in pancreatic cancer cells. Acta Pharmacol Sin 28: 1224-1230, 2007.

22. Qualtrough D, Buda A, Gaffield W, Williams AC and Paraskeva C: Hedgehog signalling in colorectal tumour cells: induction of apoptosis with cyclopamine treatment. Int J Cancer 110: 831-837, 2004.

23. Klapper LN, Kirschbaum MH, Sela M and Yarden Y: Biochemical and clinical implications of the ErbB/HER signaling network of growth factor receptors. Adv Cancer Res 77: 25-79, 2000.

24. Tortora G, Bianco R, Daniele G, et al: Overcoming resistance to molecularly targeted anticancer therapies: rational drug combinations based on EGFR and MAPK inhibition for solid tumours and haematologic malignancies. Drug Resist Updat 10: 81-100, 2007.

25. Faller BA and Burtness B: Treatment of pancreatic cancer with epidermal growth factor receptor-targeted therapy. Biologics 3 : 419-428, 2009.

26. Jimeno A, Rubio-Viqueira B, Amador ML, et al: Epidermal growth factor receptor dynamics influences response to epidermal growth factor receptor targeted agents. Cancer Res 65: 3003-3010, 2005.

27. Hill CS and Treisman R: Transcriptional regulation by extracellular signals: mechanisms and specificity. Cell 80: 199-211, 1995.

28. Bononi A, Agnoletto C, De Marchi E, et al: Protein kinases and phosphatases in the control of cell fate. Enzyme Res 2011: 329098, 2011.

29. Franke TF, Yang SI, Chan TO, et al: The protein kinase encoded by the Akt proto-oncogene is a target of the PDGF-activated phosphatidylinositol 3-kinase. Cell 81: 727-736, 1995.

30. Scheid MP, Marignani PA and Woodgett JR: Multiple phosphoinositide 3-kinase-dependent steps in activation of protein kinase B. Mol Cell Biol 22: 6247-6260, 2002.

31. Sarbassov DD, Guertin DA, Ali SM and Sabatini DM: Phosphorylation and regulation of Akt/PKB by the rictor-mTOR complex. Science 307: 1098-1101, 2005.

32. Chappell WH, Steelman LS, Long JM, et al: Ras/Raf/MEK/ERK and PI3K/PTEN/Akt/mTOR inhibitors: rationale and importance to inhibiting these pathways in human health. Oncotarget 2: 135-164, 2011.

33. Datta SR, Dudek H, Tao X, Masters S, Fu H, Gotoh Y and Greenberg ME: Akt phosphorylation of BAD couples survival signals to the cell-intrinsic death machinery. Cell 91: 231-241, 1997.

34. Brunet A, Bonni A, Zigmond MJ, et al: Akt promotes cell survival by phosphorylating and inhibiting a Forkhead transcription factor. Cell 96: 857-868, 1999.

35. Cardone MH, Roy N, Stennicke HR, et al: Regulation of cell death protease caspase- 9 by phosphorylation. Science 282 : 1318-1321, 1998. 